\title{
Beyond the computer: changing medium from digital to physical
}

Article

Accepted Version

Whyte, J. (2013) Beyond the computer: changing medium from digital to physical. Information and Organization, 23 (1). pp. 41-57. ISSN 1471-7727 doi:

https://doi.org/10.1016/j.infoandorg.2013.01.002 Available at https://centaur.reading.ac.uk/30827/

It is advisable to refer to the publisher's version if you intend to cite from the work. See Guidance on citing.

To link to this article DOI: http://dx.doi.org/10.1016/j.infoandorg.2013.01.002

Publisher: Elsevier

All outputs in CentAUR are protected by Intellectual Property Rights law, including copyright law. Copyright and IPR is retained by the creators or other copyright holders. Terms and conditions for use of this material are defined in the End User Agreement.

\section{www.reading.ac.uk/centaur}

\section{CentAUR}

Central Archive at the University of Reading

Reading's research outputs online 


\section{Beyond the Computer: Changing Medium from Digital to Physical}

Jennifer Whyte, Design Innovation Research Centre, School of Construction Management and Engineering, University of Reading, UK, j.whyte@ reading.ac.uk

Forthcoming in Information and Organization, accepted January 2013

The final, definitive version of this paper is forthcoming in Information and Organization by Taylor and Francis Ltd, All rights reserved. (C) This is the accepted version, posted by the first author on the University of Reading website under the terms of the contributor agreement. Please cite the published version and refer to it for pages, etc.

\section{Highlights}

- New theoretical understanding of visualization and materialization practices

- Analyses articulate work done to change medium from digital to physical

- Trials used to interrogate and address limits of integrated digital model.

- Role of such heterogeneous trials in resolving digital and physical data

- Implications for organizations using digital infrastructure to accomplish goals 


\section{Abstract}

How can organizations use digital infrastructure to realise physical outcomes? The design and construction of London Heathrow Terminal 5 is analysed to build new theoretical understanding of visualization and materialization practices in the transition from digital design to physical realisation. In the project studied, an integrated software solution is introduced as an infrastructure for delivery. The analyses articulate the work done to maintain this digital infrastructure and also to move designs beyond the closed world of the computer to a physical reality. In changing medium, engineers use heterogeneous trials to interrogate and address the limitations of an integrated digital model. The paper explains why such trials, which involve the reconciliation of digital and physical data through parallel and iterative forms of work, provide a robust practice for realizing goals that have physical outcomes. It argues that this practice is temporally different from, and at times in conflict with, building a comprehensive dataset within the digital medium. The paper concludes by discussing the implications for organizations that use digital infrastructures in seeking to accomplish goals in digital and physical media.

\section{Keywords:}

Sociomaterial, visual representations, digital, physical, infrastructure 


\section{Introduction}

Recent research emphasises the infrastructural nature of digital technologies within the modern workplace (Monteiro, Pollock, Hanseth, \& Williams, 2012; Pipek \& Wulf, 2009). Such research shifts the focus from the 'here and now' of local interactions to the overlapping timescales and temporal processes through which information infrastructure is developed and used, what is termed the 'long-now of infrastructure' (Ribes \& Finholt, 2009). These recent insights into "large-scale, integrated and interconnected workplace information technologies" (Monteiro, et al., 2012, p. 1) build on traditions of work on infrastructure, in information systems (e.g. Hanseth, Jacucci, Grisot, \& Aanestad, 2006; Hanseth \& Monteiro, 1997; Hanseth, Monteiro, \& Hatling, 1996; Monteiro \& Hanseth, 1996) and more broadly in sociology (Bowker \& Star, 1999; Edwards, Jackson, Bowker, \& Knobel, 2007; Hughes, 1987; Star \& Ruhleder, 1996). These traditions describe infrastructure as often taken-forgranted and only partially visible to the organizational participants that use it to accomplish their goals.

A range of digital representations are brought together and made visible through the information infrastructures that support work practices. Styhre describes how vision becomes: "intimately associated with the materialities organized to accomplish particular modes of seeing" (2010, p. 64). While scholars of information and organization vary in their definitions of sociomateriality (Leonardi, 2012), Styhre's contribution is to show how the focus on observation is one approach to operationalising this notion of 'sociomateriality' in empirical studies. This approach is important as complex organizations face difficulties in visualizing and making sense of data when faced by ambiguity (Weick, 2005). Reading Styhre's work with an interest in the infrastructural nature of digital technology raises new questions. 
This paper explores the question: "How can organizations use digital infrastructures in realizing goals that have physical outcomes?" It does so by examining the temporal practices of visualization and materialization involved in building an airport terminal, London Heathrow Terminal 5, from digital designs. The term 'digital infrastructure for delivery' is used to describe an information infrastructure used in organizational delivery processes which increases tight coupling and interdependence by integrating data. The next section reviews literature on the problematic nature of digital representation in making sense of organizational risks, in the context of this increased coupling associated with digital infrastructure, to develop the theoretical basis for understanding the empirical case. Subsequent sections describe the study methods, and then findings, which show how engineers use heterogeneous trials to interrogate and address the limitations of representations. These findings extends understanding of the modes of seeing involved in the transition from digital design to physical realisation, and the final section explores this theoretical contribution to vision as an approach to understanding interactions with digital infrastructure and thus to broader literatures on socio-materiality, sensemaking and information infrastructure.

\section{Theoretical development: Organizational risks and digital representations}

Many organizations face challenges of inter-dependent sub-units and time-dependent decision-making (Perrow, 1999 [1984]). A digital infrastructure for delivery gives organizations these characteristics. Such organizations are more efficient than linear systems in terms of design and production efficiency, as they have "less slack, less underutilized space, less tolerance of low-quality performance, and more multi-functional components" (Perrow, 1999 [1984]: p. 88). This efficiency is desirable, but brings with it increased organizational risks and the potential for catastrophic system accidents as interactions may 
not only be unexpected, but also incomprehensible for some critical period of time. To manage these risks, scholars argue that in such organizations there is a paradoxical need for both loose and tight coupling between activities (Perrow, 1999 [1984]; Weick, 1995). Tightcoupling and the centralization of decision-making allows for planning, coordinating work to achieve organizational goals and managing of future risks, yet at the same time, such centralization of decision-making can add to the risks that need to be managed within ongoing work practices. Snook's (2000) analysis of friendly fire in Iraq, for example, shows how tightly coupled systems with safeguards in place often prove unworkable locally. This occurs as "people become less and less familiar with the original tightly coupled logic of the entire operation" (Weick, 2004, p. 30).

The practices through which people make sense are particularly important in such organizations, as the social structure alone cannot provide a compelling explanation of how some such organizations achieve their stated goals while others fail (Vaughan 1999; Perrow 1999 [1984]). From a practice-based perspective, knowledge within organizations as generated through the patterns of everyday activity or 'practices' (Brown \& Duguid, 2001; Gherardi, 2009). Expertise is developed and sustained through ongoing social action, and highly dependent on the nature of the tools and contexts in which it is developed (Collins \& Evans, 2007). As Shrove et al. argue:

there is no such thing as 'just' doing. Instead, doings are performances, shaped by and constitutive of the complex relations - of materials, knowledges, norms, meanings and so on - which comprise the practice-as-entity (2007, p.13).

Knowledge practices bring together culturally situated people and technologies in ways that produce particular politics (Introna \& Hayes, 2011). The term 'heterogeneous trials' denotes a practice involving loosely coupled sets of experiments using different forms of 
representation across digital and physical media. This practice is contrasted with the tightcoupled practices associated with a digital infrastructure. Scholars in organization and technology studies discuss the heterogeneous relationships that are mobilised in all productive work and the learning that takes place in creative work (Oudshoorn \& Pinch, 2003; Rerup \& Feldman, 2011). As discussed below, the idea of heterogeneous trials, as a kind of practice involved in the transition from digital design to physical realisation, draws on literatures on sensemaking and decision-making using digital technologies; and visualization and materialization processes.

\subsection{Sensemaking and decision-making using digital technologies}

Technologies are often introduced into organizations to increase centralization and control (Thomas, 1994; Zuboff, 1988). As the world gets more inter-connected, digital innovation has led to efficiency gains (Argyres, 1999). Digital infrastructures make work visible in new ways, with repositories of information providing managers with oversight of the status of work and tracking the timing and nature of decisions.

As they change patterns of visibility and invisibility, these infrastructures change the locus and nature of decision-making within complex organizations in unanticipated, as well as anticipated, ways (Ciborra, 2004; Ciborra \& Hanseth, 2000). Ciborra (2004) highlights the 'drift' that occurs during implementation. He writes that:

Technologies and processes drift, so what one obtains at the end of the implementation process is not what the system was designed for originally. (2004: p. 
The concept of drift usefully draws attention to risk that arises because of the overlapping timescales and temporal processes through which information infrastructure is developed and used.

Kallinikos (2006) builds on Ciborra's work to argue that digital systems are characterized by the de-sequencing of time. Their use inflates the present, requiring rapid responses. This practice de-sequences temporal practices, creating a future-orientation of activities that leads to a loosening of the relationship with the past. Hence, Kallinikos claims that, paradoxically, the compression of the timescale of response leads to a proliferation of unpredictable events, making it difficult to plan.

New forms of practice are required to manage risk in complex, inter-connected, organizations supported by information technologies (Grabowski \& Roberts, 1999). While for Ciborra and Kallinikos drift and time-compression lead to risk, for Weick (1985), it is the isolated nature of working practices at the computer terminal that leads to episodes in which sensemaking breaks down, which he terms 'cosmological episodes':

People using information technologies are susceptible to cosmological episodes because they act less, compare less, socialize less, pause less, and consolidate less when they work at terminals than when they are away from them. As a result, the incidence of senselessness increases when they work with computer representations of events. (Weick, 1985, p. 56)

While digital technologies may be used to manage risk, these authors agree that their introduction and in particular the connections across information infrastructures, may also increase systemic risk, whether the source of the risk is drift, time-compression, or isolation. Their work points to a need for studies that articulate the kinds of practices that might be involved in mitigating such systemic risks. 
Kallinikos (2009), like Snook (2000), notes how a set of beliefs or model of a situation can oversimplify a complex situation, with technology reaching its limitations when it comes to uncertainty. The digital realm is closed (Edwards, 1996), or self-contained, as Weick notes:

It is the very self-contained character of the electronic cosmos that tempts people, when data make less and less sense, to retain assumptions rather than move to different orders of reasoning. [...] Reliance on a single, uncontradicted data source can give people a feeling of omniscience, but because these data are flawed in unrecognized ways they lead to nonadaptive action. (1985, p. 56-57)

In studies of scientific work, Galison (1996) argues that relationships between models and reality are not trustworthy. Where digital technologies are used represent physical phenomena, the computer may, problematically, come to 'stand, not for a tool, but for nature itself' (Galison, 1996, p. 157). The use of open, rather than closed systems is particularly important in managing the paradoxical need for both loose and tight coupling in complex, inter-dependent, organizations, as:

interactions, caused by proximity, common mode connections, or unfamiliar or unintended feedback loops, require many more probes of system conditions, and many more alterations of conditions. (Perrow, 1999 [1984], p. 82-83)

There is the potential for significant failures when the assumptions in underlying and widely distributed simulations and digital models are wrong (Cebon, 2009).

\subsection{Visualization and materialization practices}

The difficulty that organizations face in visualizing and making sense of uncertainty is well documented. Weick (2005: p.164), for example, describes how the ambiguous 
information from the blurred photographs from the launch of the Columbia Space Shuttle enabled the wrong digital models and simulations to gain organizational legitimacy as the problem became seen as a 'tile' problem. Although the Shuttle had images on board that might have revealed these false assumptions, and there were several requests within the organization for this imagery to be transferred to ground, these requests were never passed on to the Shuttle but were blocked from within the organization. Snook and Conner (2005) chart a total of three requests for imaging information and eight missed opportunities, as set out in the inquiry to the accident. Digital analyses that correctly predicted that the special shuttle would burn up on re-entry were discounted (Dunbar \& Garud, 2005), and instead faith was put in an erroneous computer representation.

It is in the context of these challenges of choosing which images on which to rely that Styhre's focus on media-laden observations becomes a relevant way to interrogate the sociomateriality of practice. Representations, across digital and physical media, are used in combination with one another and usually encountered in hybrid forms. A digital infrastructure for delivery connects and synthesises hybrid representations, combining pictures, notations and writing within the digital medium. Elkins (1999) articulates how pictures have forms, colors, shades and tones; notations are diagrams, maps, and systems of shared symbols, such as musical notation; and writing may be fully developed alphabetic or character-based spoken language. Outside of the digital infrastructure, such representations may become three dimensional, inscribed in physical media such as cardboard, plasticine, bricks, steel, etc. The digital can also play an integral part in creating, not simply representing, the physical: Østerlie et al (2012) develop the idea of a 'dual materiality' to explain how engineers involved in petroleum production can only observe the flow of liquids out of the wells through a sensors and computer equipment. 
The ambiguities that arise within representations and across media mean that: "seeing is not necessarily believing; sometimes we must believe before we can see" (Perrow, 1999 [1984], p. 9). Hence, it may be that transitions between forms of representations and media become important in knowledge work, to test assumptions and beliefs that inform the interpretation of representations within a medium. Such understanding of changing media builds on prior work on the sociology of professional vision (Goodwin, 1994), the distributed nature of social cognition (Hutchins, 1995) and the reconfiguration of professional work in the face of new media (Lanzara, 2009). In work on design, Henderson (1999) discusses the difficulties that engineers face in achieving their work through inflexible digital databases, arguing that:

Computer-driven implementation of a single right way to render graphic representations can destroy practices that are important for specific aspects of design activity. (Henderson, 1999: 8)

Here, representations are seen as instantiations of a future object (Ewenstein \& Whyte, 2009) and interaction with this "evolving object may form an active part in the collaborative process of its making”(Strandvad, 2011, p. 283). The active process of modelling thus becomes seen as part of decision making, for example in Yaneva's work on scale, which articulates the translations that architects make between models at different sizes (Yaneva, 2005).

While digital media have unintended as well as intended consequences, they are introduced into organizations to achieve instrumental ends. Hence, informed by research on sensemaking, decision-making, visualization and materialization, this study addresses how organizations use digital infrastructures in realizing goals that have physical outcomes. The theoretical contribution is to explain why heterogeneous trials provide a robust practice for 
realizing goals that have physical outcomes. The explanation emphasises the importance of iteration between different kinds of representations as designers use digital representations but also question and interrogate them, comparing and contrasting data across media to expose and address inconsistencies. The study shows how work done to move designs beyond the closed world of the computer is temporally different from, and at times in conflict with, building a comprehensive dataset within the digital medium. As discussed later in the paper, this has implications for organizations that use digital infrastructures in seeking to accomplish goals in digital and physical media.

\section{Research setting and methods}

\subsection{Research setting}

The organization of a construction project provides a good setting in which to explore pertinent organizational challenges (Boland, Lyytinen, \& Yoo, 2007); and has been of enduring interest to organizational scholars (Stinchcombe, 1959). The physical nature of the built environment that is generated, and the material (digital and non-digital) artefacts that are used in its production, have attracted scholars to investigate the role of sociomaterial practices in this context (Bresnen \& Harty, 2010). The temporary and distributed nature of work has also enabled practice-based scholars to examine and explore the translation of meanings across different epistemic communities (Gherardi \& Nicolini, 2000; Styhre, 2009).

The project discussed here was extremely complex and large ( $\$ 8 \mathrm{bn})$ airport terminal project, London Heathrow Terminal 5 which was organized into 16 major projects and 147 sub-projects; with a large supply chain and 8000 men on site at peak. At the time studied it was the largest construction project in Europe. As a long-term project, with design and engineering staff collocated at site for many years, the project has, on the one hand, some 
characteristics of a firm, such as a strong central coordinative system (March \& Simon, 1958). On the other hand, it is an amalgam of different firms, with different business models and strategic goals operating within a multi-institutional environment.

An incident on a previous project provided the stimulus for introducing new methods of working. Following the inquiry into the collapse of a tunnel into the airport in 1994, attention was drawn to inadequate documentation of design and construction. As the government and financiers looked to the airport owner and operator (the client for this project), they were keen to ensure that processes were in place to coordinate the work, to conduct it in an efficient manner and to document it. A set of protocols were developed and implemented for the use of a single database of design information. These were intended to support the practices governed by the contractual agreement, a new and innovative form of governance, the T5 agreement.

The client for the project, which owned and operated the airport, sought lessons from other sectors, particularly the automotive sector, and from lean approaches to construction. The contractual arrangements with their first tier suppliers and the project delivery methods emphasised non-adversarial partnering and continuous learning to improve performance and risk management. Rather than transfer risk on to its contractors, the client held all the risk associated with the development, allowing them to persuade the 52 main suppliers and their supply chain to work with them as an integrated team. To maintain the coherence of the organization there was also a large programme of employee engagement designed to make everyone in the organization feel part of the team. This ambition to find new ways of working also motivated the introduction of the new integrated software solution as a digital infrastructure for delivery. 


\subsection{Data collection and analysis methods}

Interest in the case was raised through a site visit and presentation by the main construction contractor in June 2005, though the author had informally discussed research with technical managers as early as 2001 . Table 1 shows the data sources that were collected across two main data collection phases.

<<Insert Table 1 here >>

Following seven hours of face-to-face meetings with senior managers, in which the author presented the proposed research on design and visualization technologies; the author was part of a team that conducted 30 semi-structured interviews ${ }^{1}$ within the construction contractor and client organizations between November 2005 and May 2006. These interviews included the project manager, his direct reports, managers of sub-projects, including the roof sub-project, and other members of the client and contractor firm, including managers in charge of other projects that had contrasting contractual arrangements. The author wrote half of the interview protocol for these interviews, with a focus on design and technology, and was involved in 20 of the 30 formal interviews, conducting three as the sole interviewer. Data from these interviews was coded by the author through an iterative process, during which transcripts were read multiple times and, at times, the author returned to listen again to the digital audio files. The coding activity was informed by reading of the related literatures and

\footnotetext{
1 The 30 interviews in phase 1 were conducted as part of a study with colleagues as described in the acknowledgements. Outside of these shared interviews, the author collected supplementary material during this phase and then conducted another phase of research as described.
} 
directed further reading. The coding schema and emerging findings were discussed extensively with colleagues.

In seeking to build theory in a contextually rich and grounded manner (Dougherty, 2002; Strauss \& Corbin, 1998), the author also went on site visits, obtained software demonstrations, had informal conversations and sought and gained access to documents such as the 'lessons-learnt' written for the contractor's internal use. The work is informed by ethnographic approaches to studies of organizational practices that obtain data by dwelling within different locations across the organization and through interviews (Czarniawska, 2004; Spradley, 1979) and where possible opportunities were taken to spend time at the site. Yet, as the first phase of data collection had started with the main project manager and interviewed down the hierarchy of managers, analysis of these formal interviews raised puzzles regarding engineers working practices. As the author became familiar with the interview data-set and started to analyse this data, there was a growing awareness of the 'scripts' that are refined and used by managers to motivate and lead their teams, with the eloquent descriptions of practice that were rehearsed by senior project managers in interviews being similar to those found in public fora, including, in some instances, online video recordings of them talking about their work. As the author was interested in technological practice, this data provided interesting insights into the work of managers, but raised, rather than resolved, questions about the reported work of employees.

In the process of analysing this data, it became difficult to resolve the diverse perspectives and understandings of digital technology that different sources of data revealed. For example, the roof sub-project was mentioned by a number of interviewees as an exemplary part of the project in which the digital infrastructure for delivery, with its central digital model for the project, had been both used and particularly beneficial. One of the senior 
managers had said: 'If you look at buildings, the roof is a fantastic example of how that integrated team comes together ...Plus SME [single model environment], absolutely. SME, roof team, single integrated team, SME working at its best.' Yet an interview with the roof sub-project manager suggested that the close team working did not rely on extensive use of this digital model. Hence, the author felt the need to go back to the field to clarify the significance and meaning of contradictory data. In the second data collection phase, further interviews were conducted by the author to interrogate the relationship between digital technologies and practice across the organization in more detail; and further supplementary material was sought and obtained from interviewees.

The strategy here was to interview people with specific responsibilities for design and delivery and/or hands-on experience of using systems, and to ask them about particular instances. Hence this data-collection focused in-depth on two contexts within the wider project: the central team involved in maintaining the single model and the roof sub-project on which it was seen by senior management to be most successfully implemented. The author went out with the chief engineer and roof sub-project manager into a working area on site to look at the roof, obtained more in-depth software demonstrations, had informal conversations and sought and gained access to key documents such as the original IT strategy for the project; and the assembly manual for the roof.

In this second phase, interview protocols were extended to ask questions that addressed the specific practices in which members of the organization engaged. For the central team the protocol questions was grouped into sections on the updating and integrity of the digital infrastructure; integration and coordination of data; production, prototyping and play; and creation of high-end visualizations. For the roof sub-project team, there were further questions grouped into sections on change and learning; visual practice and digital 
technologies, learning and performance, risks and reliability and critical interfaces. The data used in this paper comes from the second phase of data collection and the observational work and secondary data collected throughout the research, though its interpretation is informed by the wider trajectory of research including the interviews with senior managers.

While data was put into a qualitative analysis software application for ease of manipulation, the main work of coding was done in a single document, which, as listed in its contents page, contained the draft write-up (paper); current coding categories; data analysis tables; record of previous coding categories; and interview protocols. Keeping visible the decisions made across all of these separate stages of the research process (from the interview protocol to the draft text), allowed a more integrated approach to analysis, making it possible to keep in mind the links between the questions asked and the data collected; and the coding and analysis and the textual descriptions of the case in the paper. This enabled the basis for knowledge claims to be easily tracked back through the evidential chain during this phase of analysis. Hence, the sociomaterial practices through which work is accomplished were not only the empirical focus of this study, but were also a topic for reflection within the research work itself. In working between this coding and the literatures to develop the theoretical contribution of the paper, it was the contrast between the practices in the roof sub-project and the prescribed use of the digital infrastructure, which has become the most salient aspect of the dataset. Theorisation of the data set continued through the work on various drafts of this paper; with a focus on this salient contrast.

\section{Beyond the closed world of the computer toward a physical realisation}

The data show that, in building the airport roof, engineers do not trust the single source of data provided by a digital infrastructure for project delivery, but instead engage in heterogeneous trials to interrogate and address the limitations of digital representations. 
Heterogeneous trials are loosely coupled sets of experiments using different forms of representation that are used to check behaviours in digital models against the real world, to reconcile digital and physical data and to move from digital representations toward the final goal of realising a building. On the roof sub-project, this activity of making representations, at different scales and across a range of media, is seen as a fundamental part of making robust decisions in the process of designing and constructing the physical.

The engineers use the physicality of the artefact that is being delivered; as well as the tools used in its design in structuring the temporal pacing of work. For example, the decision by the roof sub-project team to develop the roof as a 'shed', reduced the interfaces with other sub-projects. This allowed the roof sub-project team to manage complexity and interdependence, concentrating on the complex organizational and technical challenge of delivering the roof without being also dependent on the decisions of other sub-project teams. Once the decision was made to allow the roof to span across the whole width of the terminal, the roof team no longer needed to have an interest in, or wait for, decisions about baggage handling systems and retail layout. This decision was critical in allowing the successful and early delivery of the roof. It was thus also critical in the sub-project becoming seen by managers as a good example of the use of the digital infrastructure for delivery.

As a central repository for all of the project data, an integrated software solution is intended to collate all design information from across the overall project, providing a 'single model environment' using a standardised set of file naming conventions, drawing numbering conventions and data naming conventions. Although, in the interviews with senior managers the roof sub-project was provided as an example of a part of the project that achieved success through using this digital infrastructure for delivery, as the sub-project manager explained: 
Clearly, whilst the single model environment was quite important to us, we were really abstracting from the single model environment the fundamental coordinates of the building, and then we were imposing onto the single model environment, the shell of the building, which others operated within. So we were quite controlling in the way that we used the single model environment.

Most of the decisions made by this team relied on their own modelling activities, and transitions across media, rather than data from the integrated software solution. Instead of modeling the entire roof structure, as envisioned in a single project model, the engineers in the roof sub-project team focus their modeling and design effort on one bay; or half a bay, in the knowledge that the engineering challenges in this are replicated along the structure. Hence, their work is not achieved through the central model and it is only later in the project, as a separate process to the visualization and materialisation of the roof, that a full geometric model of the roof is developed and then put into the central repository.

The following sub-sections examine the building and maintaining of the digital infrastructure for delivery; the practices of visualizing the roof to make design decisions; and the practices of making it real. Centralization of decision making across the project, through a digital infrastructure for delivery, is here seen as necessary, desirable and possible to coordinate and plan across sub-projects, but in moving beyond the closed world of the computer towards a physical realisation, the heterogeneous trials of the roof sub-project team become important. A centralized digital model reaches its limit in addressing uncertainty, as it collates what is already known. Hence the digital infrastructure cannot be relied on to accomplish relevant modes of seeing to visualize, make sense of uncertainty and conduct probes of system conditions where the goal has physical outcomes. 


\subsection{Building and maintaining the digital infrastructure}

The development of a digital infrastructure for delivery came about through the local activities of technical consultants employed by the client. This team of experts were brought together in the 1990s to develop the protocols for data use on the project. They developed a central repository for all the information, which would be fully controlled, so that only the latest signed-off information that was fit for use would be accessible by all the other subproject teams. A member of the original team of experts explained the challenge in their terms, and their approach:

how did you ensure that everything was managed properly, spatially coordinated, only existed once, and was correct and appropriate? And so we started to look, because of our work with CAD [computer-aided design software], go back to what we'd learnt in research and change from a document-centric method to a data-centric method, where we would share our data files between the teams.

The digital infrastructure they developed is a storage technology, a repository, or single source of information, with the associated set of standards, models and objects. The work of creating and maintaining this repository involves representing organizational structure, hierarchy and work processes in the software, through rules that can be codified and learnt. The rationale for using a single model, made available through digital infrastructure and supported by contractual arrangements that enable team work across firms, is that it has the potential to remove a major source of potential errors, allowing data about individual subsystems to be checked against and coordinated with other subsystems. This is a major change from usual practice, as data is often not shared across firms involved in construction projects. Geometry may be redrawn in the work of the different specialists, with variations in their drawings leading to costly changes and corrections when spatial conflicts arise in work on site. 
Such coordination across sub-systems and sub-projects is essential. Yet, many technical and organizational issues were experienced in the implementation of the integrated software solution as a digital infrastructure; and its use across the project proved fragile. Some issues were technical, as software ran too slowly on available hardware, and workarounds had to be introduced to deal with drawings and three-dimensional models. Yet the fragility of the digital infrastructure also reveals the fundamental differences between the static concept of a single centralized shared model of the project and the dynamic processes of decision-making on sub-projects across the project. The implementation of a single model led some actors to support the systematic accumulation of digital data towards the goal of the efficient delivery of a full digital model of the project, however for the roof sub-project team the goal was the robust delivery of physical structure, through processes that might not lead to the consistent accumulation over time of a comprehensive digital data-set.

In the initial technology strategy for the project, the benefit of the integrated software solution is described in an a-temporal manner, focusing on the benefits of the relatively complete central data-set for efficiency in later work, but failing to articulate the processes through which such data can be created, checked, validated, corrected, updated etc. The original guidance in the IT strategy suggest that teams should input comprehensive data to a good level of detail, but not 'frivolous' detail that would overwhelm hardware capability. In practice, the level of detail of data, the timing of inputting it into the model and the ownership of the model and related tasks became areas of contestation.

Soon after implementation, the original team that set up the protocols was disbanded. Having set up the protocols and provided training the work of this team was seen as complete. In their absence the use of the digital technologies, and the rationale for using them, drifted. The level of detail that sub-projects were required to provide increased, as 
additional benefits were seen to be achieved by accumulating all information, and 3D models were added to the data-set to be collated. An original technology champion described this expanding ambition to create:

The mother of all integration tools, that can take all the information from the various trade suppliers, integrate it in a magical way, which is through a 3D model. That's a load of bollocks. That doesn't work, because its nigh impossible to do that. Huge integration effort. Most of the information that maybe comes in from suppliers has probably been fudged, it's not to scale, and so the whole thing starts breaking down.

At the construction stage, this single model was largely used for reference, rather than as part of working practice of sub-projects. Sub-project teams were concerned with checking and verifying the quality of data in the database. In their ongoing practices, judgements have to be made about what data is relevant for decision-making within and across teams, and the value of using time and effort to generate this data.

The data collected in this research gives a sense of the overlapping timescales involved in the delivery of a major project, which involves an amalgam of firms with different business models and strategic goals, within which a sub-project team delivers physical airport terminal and a central technical team develops and maintains digital infrastructure to support this delivery. Though there was a growing pressure for integration of all project information, this was centralization was resisted by sub-project teams. Toward the end of the project, one of the main champions of the technological solution reflected in interview:

So I suppose in some ways [the project] actually blows away the myth of the single model and concurrent engineering because what you have is a number of models or bits of models owned by different people at different timelines. 
The technical team involved supporting the digital infrastructure at the end of the project put their faith in software development to better specifying processes and responsibilities and enable systematic accumulation of all the data through the life of the project. However, as discussed below, for engineers and designers, knowledge is embedded within people, not fully codifiable within the drawings or models - indeed at certain stages data may be thrown away or there may be deliberate breaks put between parallel teams that are working on a engineering problem to ensure robust delivery.

\subsection{Visualizing the roof}

The roof sub-project was seen as particularly effective at making decisions and delivering. In this sub-project, architects, engineers, construction managers and suppliers worked across firms in an integrated team, sharing responsibility and collaborating to solve problems. Many different kinds of models were generated and used to visualize the roof, and to actively engage in working out the design of the permanent structure and the processes of building it. In this work, the roof sub-project team resisted pressure from the client managers that championed work through the single digital model to clear up their design office by discarding physical models. The client managers felt that such models, in wax, cardboard or plasticine, did not represent the leading edge of practice and should be replaced by work in the digital medium. Yet the sub-project team described the process of making models as important in making decisions. As one of the architects related:

You can't decide as an architect until you've built a model, physical or otherwise, so there was this constant physical activity of model-making.

Different media facilitate decision making at different speeds, hence preliminary designs for nodes in the roof structure were worked out in plasticine models which were re-used a number of times as the design evolved. 
Changing medium allows decisions to be revisited and assumptions to be checked. A photograph of the design office in Figure 1, taken by an architect, shows the context in which the computer was used, with the practices around the screen revealing the iteration between digital and physical media in the design practice. Models are used that have different datastructures and make visible and focus attention on different aspects of the roof.

\section{$<<$ Insert Figure 1 >>}

It was through this ongoing process of iteratively testing solutions that decisions were made. To understand the forces in proposed roof forms, the engineer would create simple digital models using software on his laptop and discuss these with the architect:

we would chat one day and then I would knock together a quick model on my computer and the next morning we would talk about that and they'd have another idea and $\mathrm{I}[\ldots]$ did a little model on the way home and on the way in and we talked about it the next day.

There was no reliance on one single model, rather knowledge of the structure, its design and its erection was developed through interactions across different types of representations, such as the digital frame model used in the structural analysis and wind testing of the structure. At the same time as using the digital models for analysis, the engineer also emphasized the importance of having a scale model in the workspace for understanding the threedimensionality of the structure, saying that:

it's fairly difficult to see very much at a time on the screen, your perception of the complex shape is quite limited, you have to make the thing move around and then you can't quite remember what was on the other side, and it's all a jumble ... [on the physical model] ...you can perceive it more. It is sort of - it is there isn't it? - it's to do with the way we perceive things, so they were very useful. 
Hence the team used multiple media to develop and test alternative solutions: physical cardboard and plasticine models; and digital models - solid models of nodes and joints in the structure, models from wind-tunnel data and a model of a roof bay.

The focus of the team was on systematically considering what could go wrong. The engineer highlights the iterative nature of this work:

... the process of increasing sophistication of computer models, GSA structural models, meant that you start off with a very simple model and then you sort of throw that away and you build this other new one which is more sophisticated but you check to make sure that the answer you get from that is like the ones you get from that one, and we did that at least three times during the process, which ... that's the point where you often find errors when somebody starts new on the project and re-evaluates everything, has to look at it with a fresh set of eyes, sort of a critical set of eyes.

Here data is not accumulated, but rather knowledge is developed through the transitions across a wide set of increasingly detailed models; and checks are built into that process. The sceptical stance of engineers and checkers are indicated by the engineer in the phrase 'a critical set of eyes.' Within the engineering organization there was a team in another office that worked in parallel on the engineering challenges so that answers could be compared and checked.

An independent check was also employed to check all of the engineering calculations, using a practice that is more common in bridge design. This independent check articulated the wider suspicion of digital solutions and their implicit assumptions:

I think you have to step back and say, yes, it's very useful, but challenge it, constantly challenge what you see. Does it make sense? And have the ability to be able to get in and be able to check it. And that's the problem we find with third party software: you 
don't have the ability to get in and chase what you would like to check. So we tend to use our own.

The independent check worked outside of the shared modeling activities, recalculating the loadings of the structure from first principles.

\subsection{Making it real}

Such heterogeneous trials, involving reconciliation and iteration across media, were used to mitigate risk, and continued in the construction planning phase. The assembly manual states:

The roof $[\ldots]$ is one of the most challenging parts of the $[\ldots]$ programme and as such great efforts have been made to ensure its assembly is undertaken safely and with particular attention to the engineering of what has been termed the Big Lift.

This big lift involved jacking up six sections of roof, where each consists of one bay of the structure, and then putting in place the permanent structural elements that support their loads. In preparing for these lifts, heterogeneous trials were not only used in the design of the permanent structure, but also in the design of temporary works that hold the loads during assembly on site and in the construction planning in which many of the sub-assemblies were modeled and tested offsite.

As the process progressed, the fabricators became part of this iterative process of developing a solution, for example with half size models of details of the abutments made at the casting foundry at Burton-on-Trent for the team to inspect. When asked what he had learned on the project the steel fabricator said 'The importance of physical models - that was definitely a bonus.' As the architect recollects:

Architects will draw something eighteen times, only because the nineteenth time it really is right, and they were able to be part of that, integrate with that, and it never 
got to them. It never got to the point where they just said listen, we're fed up, and when it's done give us the damn drawings. They were ... they got into it, we all got into it, and they saw each, each version of the drawing.

To understand the complex geometries of the castings a specialist digital modeler was employed. He developed a model using a software tool that he was familiar with to help the designers understand the castings.

The success of this work led to his involvement in developing a model of a full bay of the structure through which further analysis and design was conducted. This digital modeler thus joined the design and construction team involved in designing the roof in 2001, working alongside the two physical model-builders that were also employed by the architect. He came to play a pivotal role in the team, not only in design but also in the construction planning. His mock-up of the erection sequence for the big-lift was used by senior managers, to show the project insurers that a suitable approach was being taken, as well as by the sub-project team. This showed how the roof was partially assembled at floor level and jacked up into place in six lifts. This digital work, though not conducted through the single model environment, was part of the reason that senior managers saw the success of this project as based on use of the digital infrastructure.

$<<$ Insert Figure 2>>

The first thing the construction manager did when he joined the team, in preparation for the six lifts, was to take the team's idea of a 'first run study' and do a full scale assembly of a part of the roof to ensure that all the pieces could be put together. Figure 2 shows the first run study and a computer simulation of an assembly. For the construction manager, and for the site engineer, the key questions about the digital model is about how it is reconciled with 
the reality of the world of concrete, steel, stone, earth and water and their physical properties and interactions on site, As the site engineer notes:

At the end of the day you've got to think, I've got to go out there and build it. Designing it's a piece of cake. Designing it with due knowledge and due recognition of potential tolerances is more difficult and putting it together out in the field is very hard 'cos things don't always work. People don't build things $100 \%$ and don't fabricate them $100 \%$ right.

Integrated software solution was more peripheral to practice of this nature because of the large amount of data that it brought in that was not relevant to the focus of sensemaking and decision making. There was a concern that digital modeling encouraged designers to design as though things worked by clockwork, rather than with regard to tolerances and coefficients of expansion. The chief engineer on site speculates about potential positive outcomes of using digital technologies, but then comes back to the challenge of making it real:

You need to build in tolerance. How the digital, how the digital world helps you, I suppose you can actually look at the effects of the tolerances and you could play with it and say, okay, whatever the tolerance of that was.

The construction manager also sees potential in digital technologies but shares the frustration at designers' general reluctance to think about material properties, such as the expansion and contraction of large structures, instead believing that you can 'just build a model and off you go.' As they discuss this, the chief engineer continues:

It's always a compromise between what is achievable and what is acceptable. You can either spend a million pound in the fab shop putting everything right or probably two million in the field putting it right or correcting it when it doesn't work. Getting people to spend a million in the first place is not easy 'cos they'll say, look, well 
everyone - you can achieve that can't you. No, I can't and it's a brave man who says, no, I can’t achieve it.

For the chief engineer the roof sub-project is the ideal compromise, where other sub-projects sought tolerances that were too tight. The good working relationships across the team enabled engineers' attention to be focused on making sense of uncertainty and probing system conditions to try and make the roof design both achievable and acceptable: never assuming everything is known in seeking to ensure that it can safely carry the loads it will experience during construction and when the airport is in operation.

\section{Discussion: heterogeneous trials}

In changing medium, the engineers use heterogeneous trials to interrogate and address the limitations of digital representations. As the construction manager, quoted above, indicates, rather than believing you can use a computer to 'just build a model and off you go' there is significant work required to test the validity of assumptions made in digital representations in moving beyond the closed world of the computer. Heterogeneous trials involve the reconciliation of digital and physical data through parallel and iterative forms of work. In the roof sub-project, a wide variety of models are used: these models have different data-structures and scales - digital models, which are viewed through the screen and rotated, are constructed in different formats, as solid models or as 2D vector frameworks; and some physical models are homogeneous and easily pliable whereas others are more articulated and at a scale at which they can be walked around. The various kinds of representations include both models, of nodes, joints, a bay and simulations, of dynamic loads and construction sequences across a variety of different media. The reliability of ideas is assessed by comparing and contrasting different representations, and checking results in parallel and iterative forms of work. 
One theoretical contribution of this analysis is to articulate how such heterogeneous trials provide a particular mode of seeing, extending Styhre's (2010) discussion of vision to articulate how visualization and materialization practices associated with realizing a digital design involve attention to the relationship between digital and physical. This relationship between digital models and physical outcomes is rendered invisible to observers focusing solely on the digital infrastructure, in which the context for work with digital representations is other digital representations. By using heterogeneous trials, engineers instead iterate between a range of representations in digital and physical media, enabling differences to become visible as they interrogate and seek to reconcile digital and physical data. They are engaged in actively choosing images on which to rely. In doing so, the roof sub-project team develops a mode of seeing that support the physical realisation of the roof.

This mode of seeing is different from that proposed by proponents of the shared digital model. For example, the team use models that draw attention to the design, structural loads and construction of one single repeatable bay, rather than the whole structure, as it is within this assembly that there are uncertainties that they need to resolve. Their focus is on iterative reconciliation of digital representations with what is known about physical conditions. Thus, in the airport project, tensions arise between these engineers' heterogeneous trials; and the practices associated with developing and maintaining the digital infrastructure which emphasise a systematic accumulation and efficient reuse of a single source of digital data. The engineers studied use digital representations extensively to design the roof. The findings concur, however, with work that suggests engineers face challenges in using inflexible databases (Henderson, 1991) and use, rather than seek to bridge, gaps between technologies (Bailey, Leonardi, \& Chong, 2010). Such findings suggest that it is to move 
beyond the closed world of the computer, that engineers use heterogeneous trials to see and solve problems.

A second theoretical contribution is the argument that such heterogeneous trials provide a robust practice for this move. It is the physical outcomes of the engineers' work that requires attention to potentially catastrophic simplification of different loads, material properties and failure modes in representations. The notion of 'heterogeneous trials' indicates the diverse, iterative and parallel use of representations to reconcile digital and physical media. Such trials are described here as robust as they allow the team to identify and recover from unexpected or exceptional findings in digital data, or conflicts between digital and physical data, through attention to multiple sources. Centralization of data in a digital model allows senior managers to have an overview of design work on the project. Yet, in realising the design, working across media is important in mitigating against the sense of omniscience which Weick (1985) points to as a danger in digital work. Trials in other media counter the drive for perfection in a digital model, which can sometimes become an end in its own right. Rather than focusing on questions internal to a single digital model, multiple trials focus engineers' attention on the assumptions and simplifications that have been made in the process of representation, the imperfections that exist in the real world in terms of manufacturing, assembly, and dynamics such as thermal expansion, and the quality level that is achievable.

On the roof sub-project, this work involved parallel teams, working on the same problems with different representations to ensure the robustness of solutions. Independent problem solving and checking reduces the chances of what Perrow (1999 [1984]) refers to as 'common mode failure', where people over-rely on a single source of data, failing to revisit assumptions and thus making decisions on the basis of visualizations that are ill-adapted to 
inform them. The integration of data is important for coordination on a project to design and construct an airport, which is a complex organizational context that involves multiple professions, firms and roles as well as many thousands of parts. Yet, while the single model centralises decision-making to deal with errors that can be foreseen, it collates what is known, and hence reaches its limit in dealing with uncertainty. It does not enable engineers to actively revisit the logic of decisions. The reliance on digital infrastructure in itself adds to the complexity of the project, with drift occurring in implementation (Ciborra, 2004; Kallinikos, 2006) as the single model environment, becomes treated as ends rather than a means. On each sub-project, interaction with the digital infrastructure needs to be situated within the ongoing flow of everyday practice. To retain a focus on the exercise of professional judgement, rather than rapid digital responses, the practices within the roof subproject are loosely coupled with the digital infrastructure, with members of the team have diverse interactions with digital models, depending on their roles, and a modelling expert as one member of the team. The single model is not updated until after decisions are made.

Thus, while the roof sub-project is seen as highly successful, it achieves the project goals, which motivated the introduction of the integrated digital infrastructure, through visualization and materialization practices in which it plays only a peripheral role. The decision to make the roof as a 'shed' limits the interdependencies with other sub-projects and allows the roof sub-project team to work in an integrated way, bringing together engineering, design, construction, manufacturing and digital modelling knowledge in regular meetings. This decision also reduces the need to refer to data in the single model, which integrates design data across the whole project. A 'dual materiality' is at play here, not as it is in Østerlie's (2012) study of petroleum extraction, where the existing physical arrangements are always observed through digital media, but instead as the proposed design of a future 
physical structure shapes, as well as is shaped by, the teams' configuration and use of representations. This dual materiality affects the temporal pacing of work. By reducing the interdependence between their part of the airport terminal and other sub-projects, the team are able to plan delivery, and become seen as a highly successful sub-project. They are freed from the tight coupling of their design decisions with those that need to be made on other sub-projects, such as baggage handling and retail and from the need to see these interdependencies in a single model.

The findings have implications for recent debates on sociomateriality as they examine and articulate the nature of the work to move between digital and physical representations. This builds on and furthers Styhre's discussion of media-laden observation. Scholars in information and organization have diverse understandings of sociomateriality. While scholars have focused on the materiality of digital media (Scott \& Orlikowski, 2012) or digital representations (Dourish \& Mazmanian, 2011); others discuss their apparent 'immateriality' or differences from physical objects (Yoo, 2012); and still others use the term materiality to indicate physical phenomena (Robey, Schwang, \& Jin, 2003), or to discuss the physical context of digital work (Harty \& Whyte, 2010). By answering Styhre's call for further research on visualizing and materializing, this research examines the organizational practices involved in changing medium from digital to physical artifacts in the pursuit of organizational goals. The idea of heterogeneous trials draws attention to the differences between physical and digital materialities, expanding previous work on their interrelationships (Østerlie, et al., 2012) to show how a proposed physical artefact shapes and is shaped by work with digital representations. The findings concur with recent work on sensemaking that showing how reliable IT systems can promote mindlessness (Butler \& Gray, 2006), and how mindful and mindless activities happen together (Carlo, Lyytinen, \& 
Boland, 2012), extending this debate by suggesting the explanatory power of the modes of seeing enabled by particular practices in understanding what organizations are mindful about and to explain their ability to manage risk and develop reliable outcomes.

The findings also have implications for understandings of infrastructure, and particularly the temporal questions associated with its implementation and use. The analyses suggest that research needs to conceptualize digital infrastructures as always incomplete and, at overlapping timescales, in development. From such a perspective, digital infrastructure for delivery is inherently fragile. This suggests new approaches to research that do not see it as useful to, as the practitioners in this case did, frame fragility as being the result of technology immaturity or issues of implementation. A part of the incompleteness of digital infrastructure is its inability to fully contain representations of the physical world. Thus, while some challenges may be related to the maturity of the technology, other challenges engineers face, first noted in Henderson's (1991) observations of engineers using inflexible databases, are intrinsic to the nature of digitally-enabled work where the goal is to develop physical outcomes. A further challenge is the relationship between the long now of infrastructure (Monteiro, et al., 2012) and the expanded present (Kallinikos, 2006) that is experienced by those that use it and try to integrate it into their practice.

In order to use digital infrastructures in realizing goals that have physical outcomes, organizations must support robust visualization and materialization practices. These practices will be different from those required to achieve goals that can be accomplished within a digital infrastructure. Engineering work may continue to be further digitised and integrated through increasingly sophisticated digital infrastructures, but there is a need to create open systems in which systemic risks can be mitigated by comparing and contrasting across the digital and physical. This research articulates how IT developers and champions thus face the 
challenge of creating open systems in which an evolving and partial digital infrastructure can be used to achieve goals beyond the computer.

\section{Acknowledgements}

The author gratefully acknowledges the support of colleagues, Tim Brady, Catelijne Coopmans, Andrew Davies and David Gann, with whom she worked in the first phase of her data collection; Rogers, Stirk, Harbour + Partners for permission to reproduce images; and UK funders, EPSRC and ESRC through grants EP/E001645/1, RES-331-27-0076 and $\mathrm{EP} / \mathrm{H} 02204 \mathrm{X} / 1$ at the University of Reading, and previous support at Imperial College Business School.

\section{References}

Argyres, N. S. (1999). The impact of information technology on coordination: evidence from the B-2 "Stealth" Bomber. Organization Science, 10(2), 162-179.

Bailey, D. E., Leonardi, P. M., \& Chong, J. (2010). Minding the Gaps: Understanding Technology Interdependence and Coordination in Knowledge Work Organization Science, 21(3), 713-730.

Boland, R. J., Lyytinen, K., \& Yoo, Y. (2007). Wakes of Innovation in Project Networks: The Case of Digital 3-D Representations in Architecture, Engineering, and Construction. Organization Science, 18(4), 631-647.

Bowker, G., \& Star, S. L. (1999). Sorting Things Out. Cambridge, MA: MIT Press.

Bresnen, M., \& Harty, C. (2010). Editorial: Special Issue on Objects, Knowledge Sharing and Knowledge Transformation in Projects. Construction Management and Economics, 28(6), 549-555. 
Brown, J. S., \& Duguid, P. (2001). Knowledge and organization: a social-practice perspective. Organization Science, 12(2), 198-213.

Butler, B. S., \& Gray, P. H. (2006). Reliability, Mindfulness and Information Systems. MIS Quarterly, 30(2), 211-224.

Carlo, J. L., Lyytinen, K., \& Boland, R. J. (2012). Dialectics of Collective Minding: Contradictory Appropriations of Information Technology in a High-Risk Project. MIS Quarterly, 36, 1081-1108.

Cebon, P. (2009). Innovating Our Way to a Meltdown. MIT Sloan Management Review, $50(2), 13-15$.

Ciborra, C. (2004). Digital Technologies and the Duality of Risk: LSE Discussion Paper 24.

Ciborra, C., \& Hanseth, O. (2000). Introduction: from control to drift. In C. Ciborra \& associates (Eds.), From Control to Drift: the Dynamics of Corporate Information Infrastructures. Oxford: Oxford University Press.

Collins, H., \& Evans, R. (2007). Rethinking expertise. Chicago: The University of Chicago Press.

Czarniawska, B. (2004). On Time, Space, and Action Nets. Organization, 11(6), 773-791.

Dougherty, D. (2002). Building grounded theory: Some principles and practices. In J. A. C. Baum (Ed.), Blackwell companion to organizations (pp. 849-867). Oxford Blackwell Publishers.

Dourish, P., \& Mazmanian, M. (2011). Media as Material: Information Representations as Material Foundations for Organizational Practice. Paper presented at the Working Paper for the Third International Symposium on Process Organization Studies, Corfu, Greece. 
Dunbar, R., \& Garud, R. (2005). Data indeterminancy: One NASA, two modes. In W. H. Starbuck \& M. Farjoun (Eds.), Organization at the Limit: Lessons from the Columbia Disaster (pp. 202-219). Malden, MA: Blackwell Publishing.

Edwards, P. N. (1996). The closed world: computers and the politics of discourse in cold war America. Cambridge, MA: MIT Press.

Edwards, P. N., Jackson, S. J., Bowker, G. C., \& Knobel, C. P. (2007). Understanding Infrastructure: Dynamics, Tensions, and Design. Ann Arbor: DeepBlue.

Elkins, J. (1999). The Domain of Images. Ithaca: Cornell University Press.

Ewenstein, B., \& Whyte, J. (2009). Knowledge practices in design: The role of visual representations as 'epistemic objects'. Organization Studies, 30(1), 7-30.

Galison, P. (1996). Computer simulations and the trading zone. In P. Galison \& D. J. Stump (Eds.), The disunity of science. Stanford: Stanford University Press.

Gherardi, S. (2009). Introduction: The Critical Power of the 'Practice Lens'. Management Learning, 40, 115-128.

Gherardi, S., \& Nicolini, D. (2000). To Transfer is to Transform: The Circulation of Safety Knowledge. Organization, 7(2), 329-348.

Goodwin, C. (1994). Professional vision. American Anthropologist, 96(3), 606-633.

Grabowski, M., \& Roberts, K. (1999). Risk mitigation in virtual organizations. Organization Science, 11, 704-721.

Hanseth, O., Jacucci, E., Grisot, M., \& Aanestad, M. (2006). Reflexive Standardization: SideEffects and Complexity in Standard Making. MIS Quarterly, Special Issue on Standard Making.

Hanseth, O., \& Monteiro, E. (1997). Inscribing behavior in information infrastructure standards. Accounting, Management \& Information Technology, 7(4), 183-211. 
Hanseth, O., Monteiro, E., \& Hatling, M. (1996). Developing information infrastructure: The tension between standardization and flexibility. Science, Technology and Human Values, 21(4), 407-426.

Harty, C., \& Whyte, J. (2010). Emerging hybrid practices in construction design work: the role of mixed media Journal of Construction Engineering and Management, 136(4), 468-476.

Henderson, K. (1991). Flexible sketches and inflexible data bases: visual communication, conscription devices, and boundary objects in design engineering. Science, Technology and Human Values, 16(4), 448-473.

Henderson, K. (1999). On Line and On Paper: Visual representations, visual culture and computer graphics in design engineering. Cambridge, MA: MIT Press.

Hughes, T. P. (1987). The evolution of large technical systems. In T. P. H. W.E. Bijker, and T. Pinch (Ed.), The social construction of technological systems. Cambridge, MA: MIT Press.

Hutchins, E. (1995). Cognition in the Wild. Cambridge, MA: MIT Press.

Introna, L. D., \& Hayes, N. (2011). On sociomaterial imbrications: What plagiarism detection systems reveal and why it matters. Information and Organization, 21, 107-122.

Kallinikos, J. (2006). The consequences of information: institutional implications of technological change. Cheltenham: Edward Elgar.

Kallinikos, J. (2009). On the computational rendition of reality: artefacts and human agency. Organization, 16, 183-202.

Lanzara, G. F. (2009). Reshaping Practice Across Media: Material Mediation, Medium Specificity and Practical Knowledge in Judicial Work. Organization Studies, 30(12), 1369-1390. 
Leonardi, P. (2012). Materiality, Sociomateriality, and Socio-Technical Systems: What Do These Terms Mean? How Are They Different? Do We Need Them? In P. Leonardi, J. Kallinikos \& B. Nardi (Eds.), Materiality and Organizing: Social Interaction in a Technological World. Oxford: Oxford University Press.

March, J. G., \& Simon, H. A. (1958). Organizations: Wiley.

Monteiro, E., Pollock, N., Hanseth, O., \& Williams, R. (2012). From artefacts to infrastructures. Computer Supported Cooperative Work.

Monteiro, L., \& Hanseth, O. (1996). Social shaping of information infrastructure: on being specific about the technology. In W. J. Orlikowski, M. Jones \& J. DeGross (Eds.), Information technology and changes in organizational work (pp. 325-348). London: Chapman Hall.

Østerlie, T., Almklov, P. G., \& Heps $\varnothing$, V. (2012). Dual materiality and knowing in petroleum production. Information and Organization, 22, 85-105.

Oudshoorn, N., \& Pinch, T. (2003). How Users Matter: The Co-Construction of Users and Technologies. Cambridge, MA: MIT Press.

Perrow, C. (1999 [1984]). Normal Accidents: Living with High-Risk Technologies. Princeton, New Jersey: Princeton University Press.

Pipek, V., \& Wulf, V. (2009). Infrastructuring: Toward an Integrated Perspective on the Design and Use of Information Technology. Journal of the Association for Information Systems, 10(5), 447-473.

Rerup, C., \& Feldman, M. (2011). Routines as a source of change in organizational schemata: the role of trial-and-error learning. Academy of Management Journal, 54 (3), 577-610.

Ribes, D., \& Finholt, T. A. (2009). The Long Now of Technology Infrastructure: Articulating Tensions in Development. Journal of the Association for Information Systems, 10(5). 
Robey, D., Schwang, K. S., \& Jin, L. (2003). Intertwining material and virtual work. Information and Organization, 13, 111-129.

Scott, S. V., \& Orlikowski, W. J. (2012). Great Expectations:The Materiality of Commensurability in Social Media. In P. Leonardi, J. Kallinikos \& B. Nardi (Eds.), Materiality and Organizing: Social Interaction in a Technological World. Oxford: Oxford University Press.

Shrove, E., Watson, M., Hand, M., \& Ingram, J. (2007). The design of everyday life. Oxford: Berg.

Snook, S. A. (2000). Friendly Fire: The Accidental Shootdown of US Black Hawks over Northern Iraq Princeton University Press.

Snook, S. A., \& Conner, J., C. (2005). The Price of Progress: Structurally induced inaction. In W. H. Starbuck \& M. Farjoun (Eds.), Organization at the Limit, Lessons from the Columbia Disaster (pp. 178-201). Malden, MA: Blackwell.

Spradley, J. (1979). The Ethnographic Interview: Thomas Learning.

Star, S. L., \& Ruhleder, K. (1996). Steps Toward an Ecology of Infrastructure: Design and Access for Large Information Spaces. Information Systems Research, 7(7), 111-134.

Stinchcombe, A. L. (1959). Bureaucratic and Craft Administration of Production: A Comparative Study. Administrative Science Quarterly, 4(2), 168-187.

Strandvad, S. M. (2011). Materializing ideas: A socio-material perspective on the organizing of cultural production. European Journal of Cultural Studies, 14(3), 283-297.

Strauss, A., \& Corbin, J. (1998). Basics of Qualitative Research: Techniques and Procedures for Developing Grounded Theory. Thousand Oaks, CA: Sage.

Styhre, A. (2009). Managing Knowledge in the Construction Industry. London and New York: Spon Press. 
Styhre, A. (2010). Organizing technologies of vision: Making the invisible visible in medialaden observations. Information and Organization, 20(1), 64-78.

Thomas, R. J. (1994). What Machines Can't Do: Politics and Technology in the Industrial Enterprise. Berkeley and Los Angeles, CA: University of California Press.

Weick, K. E. (1985). Cosmos versus chaos: sense and nonsense in electronic contexts. Organizational Dynamics, 14(2), 51-64.

Weick, K. E. (1995). Sensemaking in Organizations. London: Sage Publications.

Weick, K. E. (2004). Normal Accident Theory As Frame, Link and Provocation. Organization \& Environment, 17, 27-31.

Weick, K. E. (2005). Making Sense of Blurred Images: Mindful Organizing in Mission STS107. In W. Starbuck \& M. Farjoun (Eds.), Organization at the Limit: Lessons from the Columbia Disaster (pp. 159-177). Malden, MA: Blackwell.

Yaneva, A. (2005). Scaling Up and Down: Extraction Trials in Architectural Design. Social Studies of Science, 35(6), 867-894.

Yoo, Y. (2012). Digital Materiality and the Emergence of an Evolutionary Science of the Artificial. In P. Leonardi, J. Kallinikos \& B. Nardi (Eds.), Materiality and Organizing: Social Interaction in a Technological World. Oxford: Oxford University Press.

Zuboff, S. (1988). In the Age of the Smart Machine: The future of work and power. Basic Books: New York. 


\begin{tabular}{|c|c|c|c|c|c|c|c|}
\hline & \multirow{3}{*}{$\begin{array}{l}\text { Observation } \\
\text { (days) }\end{array}$} & \multicolumn{5}{|c|}{ Interviews } & \multirow{3}{*}{$\begin{array}{l}\text { Primary } \\
\text { Source } \\
\text { Materia } \\
\text { (pages) }\end{array}$} \\
\hline & & \multicolumn{2}{|l|}{ Client } & \multicolumn{2}{|c|}{ Supply chain } & \multirow[t]{2}{*}{ Total } & \\
\hline & & Formal & Informal & Formal & Informal & & \\
\hline $\begin{array}{l}\text { First phase data } \\
\text { collection }\end{array}$ & 3.5 & 15 & 8 & 15 & 24 & 62 & 198 \\
\hline $\begin{array}{l}\text { Second phase data } \\
\text { collection }\end{array}$ & 0.5 & & & 17 & 8 & 25 & 426 \\
\hline Totals & 4 & & & & & 87 & 624 \\
\hline
\end{tabular}

Table 1: Sources of data used in the analysis 
Figure 1: The computer screen and the practices of the design office

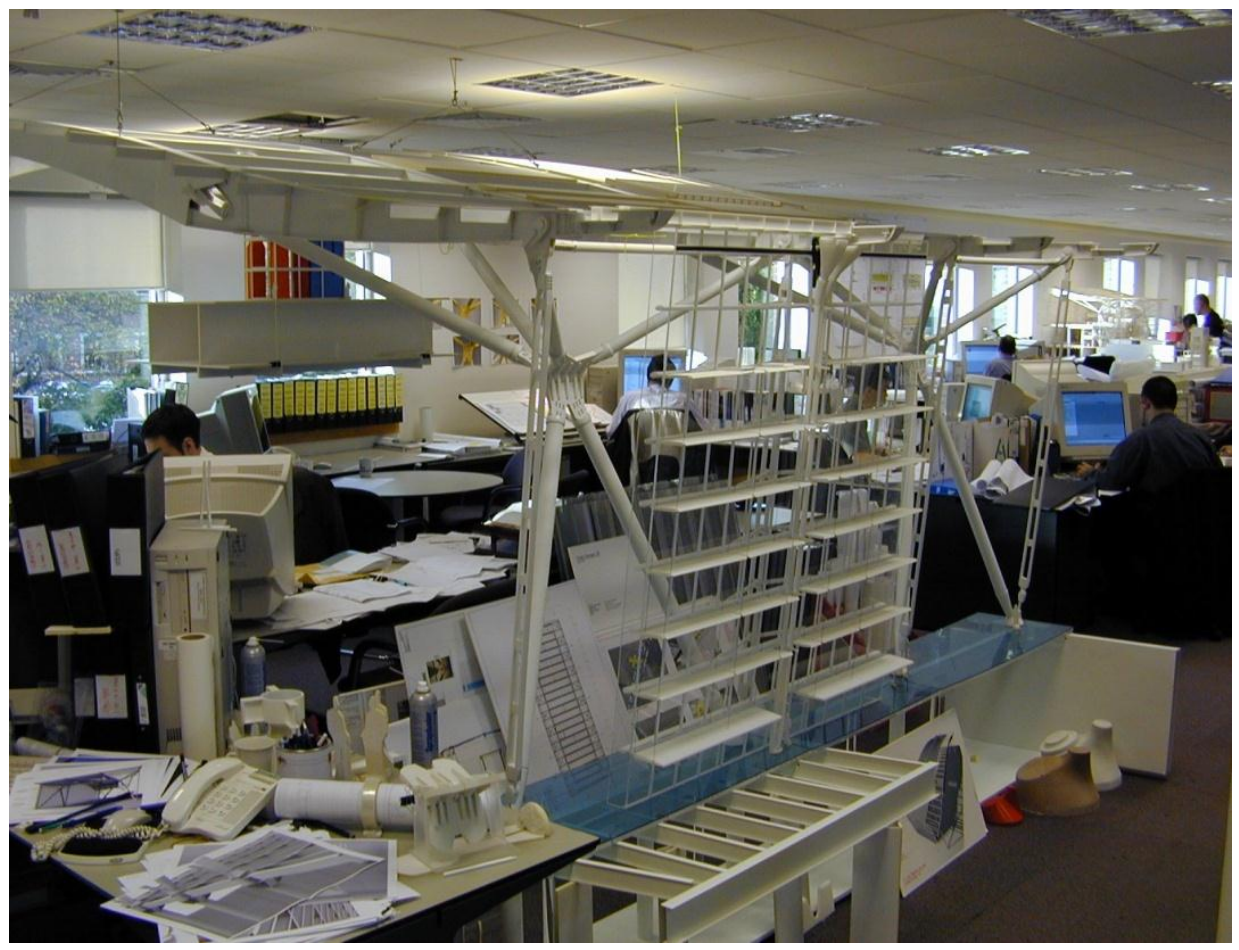


Figure 2: The 'first-run' study and a computer model of assembly
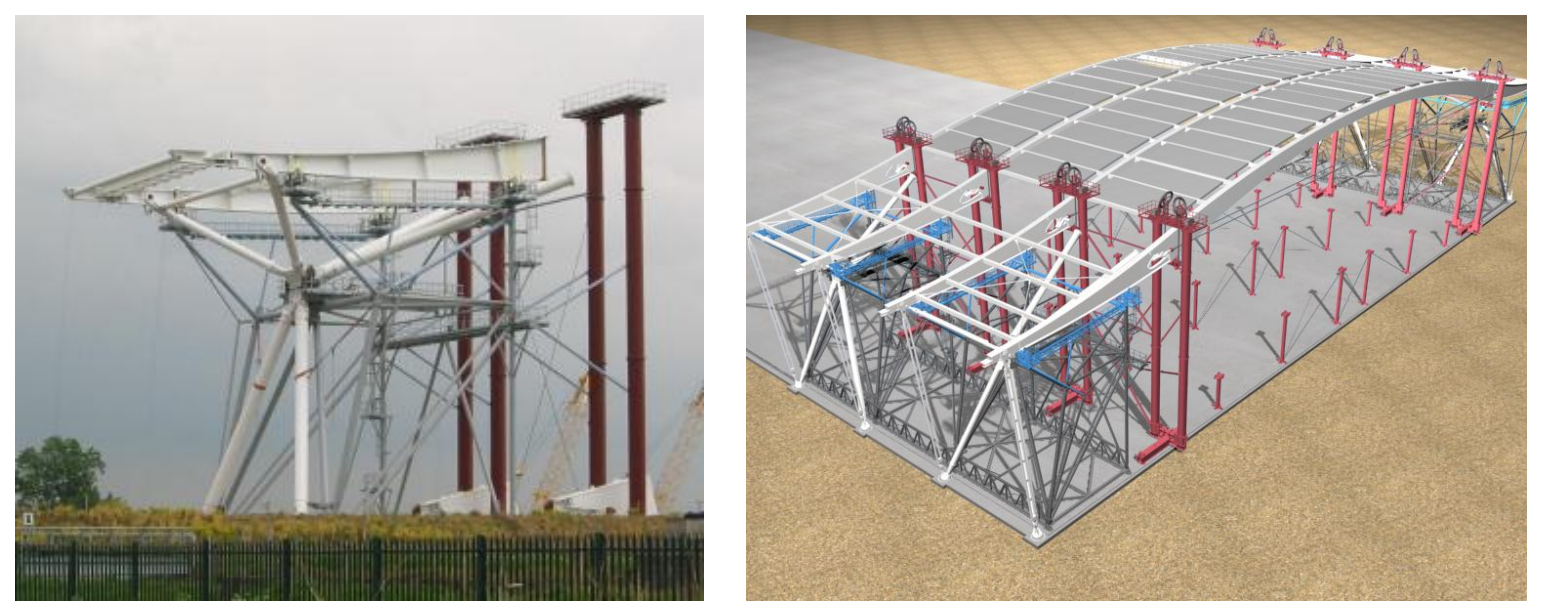